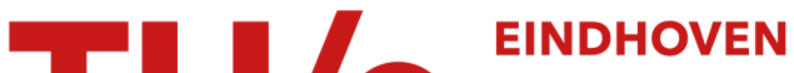 UNIVERSITY OF TECHNOLOGY
}

\section{Efficient Brownian dynamics simulation of particles near walls. I. Reflecting and absorbing walls}

\section{Citation for published version (APA):}

Peters, E. A. J. F., \& Barenbrug, T. M. A. O. M. (2002). Efficient Brownian dynamics simulation of particles near walls. I. Reflecting and absorbing walls. Physical Review E - Statistical, Nonlinear, and Soft Matter Physics, 66(5), 056701-1/7. [056701]. https://doi.org/10.1103/PhysRevE.66.056701

DOI:

10.1103/PhysRevE.66.056701

Document status and date:

Published: 01/01/2002

\section{Document Version:}

Publisher's PDF, also known as Version of Record (includes final page, issue and volume numbers)

\section{Please check the document version of this publication:}

- A submitted manuscript is the version of the article upon submission and before peer-review. There can be important differences between the submitted version and the official published version of record. People interested in the research are advised to contact the author for the final version of the publication, or visit the $\mathrm{DOI}$ to the publisher's website.

- The final author version and the galley proof are versions of the publication after peer review.

- The final published version features the final layout of the paper including the volume, issue and page numbers.

Link to publication

\section{General rights}

Copyright and moral rights for the publications made accessible in the public portal are retained by the authors and/or other copyright owners and it is a condition of accessing publications that users recognise and abide by the legal requirements associated with these rights.

- Users may download and print one copy of any publication from the public portal for the purpose of private study or research.

- You may not further distribute the material or use it for any profit-making activity or commercial gain

- You may freely distribute the URL identifying the publication in the public portal.

If the publication is distributed under the terms of Article 25fa of the Dutch Copyright Act, indicated by the "Taverne" license above, please follow below link for the End User Agreement:

www.tue.nl/taverne

Take down policy

If you believe that this document breaches copyright please contact us at:

openaccess@tue.nl

providing details and we will investigate your claim. 


\title{
Efficient Brownian dynamics simulation of particles near walls. I. Reflecting and absorbing walls
}

\author{
E. A. J. F. Peters* \\ Department of Chemical Engineering, Universiteit van Amsterdam, Nieuwe Achtergracht 166, 1018WV Amsterdam, The Netherlands \\ Th. M. A. O. M. Barenbrug \\ Department of Chemical Engineering, Università degli Studi di Napoli Frederico II, Piazzale V. Tecchio 80, 80125 Napoli, Italy
}

(Received 5 June 2002; published 6 November 2002)

\begin{abstract}
In this paper a method of numerically handling boundary conditions within Brownian dynamics simulations is discussed. The usual naive treatment of identifying reflection or absorption processes by checking for boundary crossings yields $O(\sqrt{\Delta t})$ discretization errors. The method we propose here yields $O(\Delta t)$ errors, similar to the case of Brownian dynamics without wall interaction. The main idea is to ensure that the zeroth (in the case of absorption), first, and second moments of the particle's displacement steps are correct up to order $\Delta t$. To fulfill this requirement near a wall, one has to include nontrivial corrections, because the stochastic contribution does not average out when the distance to the wall is of the order of the step length. We demonstrate here that the method substantially reduces the discretization error for the simple cases of an absorbing and a reflecting wall. Our method comprises an improvement over earlier methods proposed by Lamm and Schulten [J. Chem. Phys. 78, 2713 (1983)] and Öttinger [J. Chem. Phys. 91, 6455 (1937)]. Their methods heavily depend on full, explicit, analytical expressions for solutions of the diffusion equation near a wall, which they use to make a correction after a stochastic step has been made. Our method only involves the, usually much simpler, lowest moments (up to the second) of the probability density distributions for the displacement of the particle in one time step. This means the method only uses the initial particle position to determine a valid step, and there is no need for corrections afterwards. Because much less information is needed (three moments instead of full probability densities), in many cases information can be stored simply in interpolation functions and there is no need to evaluate complicated analytical expressions at every time step. This makes the method more efficient and easy to generalize to other situations than the relatively simple case of a flat wall. Moreover, because analytic expressions are not needed, other methods to determine the needed moments can be used. This makes our method much more flexible.
\end{abstract}

DOI: 10.1103/PhysRevE.66.056701

PACS number(s): 02.70.Ns, 05.40.-a, 02.50.Ey

\section{INTRODUCTION}

If a mesoscopic particle is submerged in a heat bath of a very large number of much smaller particles (usually of a molecular size), which undergo frequent, numerous collisions with the larger particle, the former exhibits a quite irregular type of motion, called Brownian motion. The motion of relatively large molecules, e.g., polymers and proteins, is usually assumed to fall in the same category.

Brownian motion can be modeled by random stochastic fluctuating contributions to the displacement vector of a particle, thus discarding the detailed physics of the collisions of neighboring particles. In many systems the net effect of such collisions is a local thermal equilibration in momentum space. If this is the case, and if one is not interested in the details of the equilibration process itself, idealized Brownian motion is a useful description. Especially from the point of view of numerical simulation, there are large benefits in using a Brownian description instead of a more detailed one. In the detailed description the time scales of collisions are very small, i.e., molecular time scales, requiring very small numerical time steps. This makes it virtually impossible to simulate processes up to a macroscopic time scale regime by using methods such as molecular dynamics. In more coarse-

*Electronic address: fpeters@science.uva.nl grained methods such as Brownian dynamics, these very small time scales are discarded and the gap between simulation time steps and macroscopic times can be bridged.

Generally, one describes Brownian motion in two equivalent, but essentially different ways. One of these descriptions uses stochastic differential equations (SDEs), also called Langevin equations. The SDE for the motion of a Brownian particle contains two contributions. The first contribution contains the deterministic, nonstochastic change in the position of a particle during a time step. The second one, which results from the frequent, brief impacts of the small particles, is modeled by a stochastic process with correlation time zero, in agreement with the large time scale of the description itself. The numerical method of solving SDEs is called Brownian dynamics. The method consists of the simulation of a large number of independent realizations of the deterministic and stochastic processes acting on the particle. Macroscopic quantities, e.g., the behavior of a large collection of Brownian particles, are then obtained by performing an ensemble average over these realizations.

The other description focuses on the time evolution of the spatial distribution function of the particles. Such an evolution equation is generally called a "Fokker-Planck" or "advection-diffusion" equation. Besides the fact that the SDE gives a more intuitive description of the motion of the particle, in many cases the numerical method used to solve this equation is much cheaper in terms of computational re- 
sources than that for solving the corresponding FokkerPlanck equation. In this paper we (therefore) focus on the former.

The one-dimensional SDE for the position $X$ of the Brownian particle, having a constant diffusivity $D$, has the form

$$
d X=A(X) d t+\sqrt{2 D} d W .
$$

The first term at the right-hand side of Eq. (1) is the deterministic part. The second term is the stochastic part. The stochastic process $W$ in this term is the so-called "Wiener process." This process is a Gaussian process with realizations (or paths) that are not differentiable in time. Because of the nondifferentiability of the Wiener process, the stochastic differential equation as given by Eq. (1) is meaningless in a strict formal sense. It is shorthand for a Riemann sum (in the limit of $\Delta t \rightarrow 0)$. An increment in the Riemann sum has the form

$$
\Delta X=A(X) \Delta t+\sqrt{2 D} \Delta W .
$$

This expression encompasses the simplest first-order (Euler forward) time discretization of Eq. (1). Because of the nature of the Wiener process one has to agree upon where to evaluate the (stochastic) integrand within a time interval (for the case with a constant diffusion coefficient this is not relevant). We will use the Ito interpretation, which means the evaluation of the integrand at the start of every time interval.

The increments of the Wiener process are mutually independent and have expectation value zero,

$$
\langle\Delta W\rangle=0 .
$$

Due to this property the expectation value of an increment is given by

$$
\lim _{\Delta t \rightarrow 0} \frac{\langle\Delta X\rangle}{\Delta t}=\langle A(X)\rangle
$$

Therefore $A(X)$ can be interpreted as a drift velocity. The square of an increment of the Wiener process is proportional to time

$$
\left\langle(\Delta W)^{2}\right\rangle=\Delta t .
$$

For the increment of $X$ this gives

$$
\lim _{\Delta t \rightarrow 0} \frac{\left\langle(\Delta X-\langle\Delta X\rangle)^{2}\right\rangle}{\Delta t}=2 D,
$$

i.e., the variance of the displacements is proportional to the time step. We conclude that, if we would construct the particle's displacement probability distribution after one time step, the first moment of that distribution would be equal to $A \Delta t$ and the second moment would be $2 D \Delta t$.

Vice versa, it is well known, that in the first-order numerical approximation Eq. (2), one does not need to use a Gaussian distributed Wiener increment. In fact any (nonpathological) distribution $\Delta U$, which has the correct first and second moments (i.e., 0 and $\Delta t$ ), will do. This is a consequence of a generalized central limit theorem. (If a higher-order discretization is desired, also higher-order moments need to be considered.) The simple observation that apparently only the zeroth, first, and second moments are relevant for a firstorder discretization is the main postulate of this paper, and forms the basis for our simulation method.

In many "naive" Brownian dynamics implementations this "requirement of correct moments" is fulfilled fortuitously. However, in some situations the wildly fluctuating character of the Brownian motion can give rise to large discretization errors. An important example of such a situation is interaction with a wall. When considering the positions before and after a stochastic displacement of a particle in the vicinity of a wall, one might conclude that the wall is not hit and consequently there was no wall interaction. However, the Brownian path between the starting and the final position is not a straight line, but a random walk. This means that wall interaction is quite likely to have occurred somewhere within the time step, although the starting and final positions are on the same side of the wall. If this fact is not taken into account, large discretization errors are the result. In the following we explain the source of this error and present a fairly general method to resolve this problem.

\section{DISCRETIZATION NEAR A WALL}

The first-order discretization given by Eq. (2), is a valid discretization, with an accuracy of $O(\Delta t)$, only as long as the step size $\Delta X$ is much smaller than all physical relevant length scales in the system. However, when a particle approaches a wall, another relevant length scale emerges, namely, the distance to that wall. For small distances to the wall, Eq. (2) is not a valid discretization of Eq. (1), and the use of Eq. (2) will give large discretization errors. The simplest approach to tackle this problem would be to decrease the time step when approaching the wall, until the discretization error would have become small enough. Clearly, due to the slowly decreasing error, this method is inefficient.

Generally, one would prefer to use a constant time step, and not be restricted by the relatively rare event of wall interaction. This means that one could also attempt to correct the discretization, Eq. (2), for the presence of the wall. This can be done in a straightforward manner.

For the discretization of an SDE in free space, the expectation value of the displacement is $O(\Delta t)$. The $O(\sqrt{\Delta t})$ contribution of the stochastic part averages out because of the isotropy of the Wiener process. Of course, when a wall is present, displacements beyond the wall are not allowed. This means that, close to the wall, where the average displacement is relatively large compared to the distance to the wall, the $O(\sqrt{\Delta t})$ stochastic contributions to the displacement can no longer be isotropically distributed, and must lead to a net contribution to the motion, directed away from the wall. Of course, the scaling of this extra contribution is the same as of its constituent steps, $O(\sqrt{\Delta t})$. Omission of this contribution in the discretization of the SDE will therefore lead to an error of the same order; the error indeed obtained using the "naive" method. First we propose a way to determine this extra 
contribution near the wall. At the same time this enables us to address the nature of the simulation error in more detail. Then we apply our method to two cases: a fully absorbing, and a fully reflecting wall.

For any chosen time step, the possible displacements of the Brownian particle associated with this time step are described by some spatial distribution function. To approximate this displacement probability distribution by a stochastic differential equation, we postulate that the discretization should be such that the zeroth, first, and second moments of the displacement probability distribution are predicted correctly. This conjecture can be proved by monitoring the change of expectation values of functions of the dynamical variables. If the change of the expectation value of any function is predicted correctly within a certain order of $\Delta t$, then the algorithm is said to be accurate (in the weak sense) with this same order. For the sake of simplicity we will consider the case of constant zeroth moment in the proof.

Then change of the expectation value of $f(X)$ after a time step $\Delta t$ can be approximated by a Taylor series,

$$
\Delta\langle f(X)\rangle=\left\langle f^{\prime}\left(X_{0}\right) \Delta X\right\rangle+\frac{1}{2}\left\langle f^{\prime \prime}\left(X_{0}\right)[\Delta X]^{2}\right\rangle+\left\langle O\left([\Delta X]^{3}\right)\right\rangle .
$$

Within the average one can compute the conditional expectation values of the moments of $\Delta X$ for fixed $X_{0}$ for every $X_{0}$ first (denoted by $\langle\cdots\rangle_{X_{0}}$ ), and perform the average over $X_{0}$ later,

$$
\begin{aligned}
\Delta\langle f(X)\rangle= & \left\langle f^{\prime}\left(X_{0}\right)\langle\Delta X\rangle_{X_{0}}\right\rangle+\frac{1}{2}\left\langle f^{\prime \prime}\left(X_{0}\right)\left\langle[\Delta X]^{2}\right\rangle_{X_{0}}\right\rangle \\
& +O\left(\left\langle[\Delta X]^{3}\right\rangle_{X_{0}}\right) .
\end{aligned}
$$

Because stochastic differential equations give rise to a Markov process, these conditional probabilities can be expressed as functions of $X_{0}$ only. Within our algorithm the lower-order moments of the conditional displacements are simulated correctly. This means that, if $X_{0}$ is sampled correctly, the first two terms on the right-hand side are exact. Therefore the leading order of the error will be $\left\langle O\left([\Delta X]^{3}\right)\right\rangle_{X_{0}}$. Near the wall this will give an $O\left([\Delta t]^{3 / 2}\right)$ discretization error. One might think that the global discretization error will be $O\left([\Delta t]^{1 / 2}\right)$, because there are a number of $O(1 / \Delta t)$ displacements with an $O\left([\Delta t]^{3 / 2}\right)$ contribution to the error. This is not true. The reason is that in the bulk the odd-power contributions of the displacements will give zero contributions to the expectation value, and the leading error will be given by the fourth power of $\Delta X$ (second power in $\Delta t$ ). Furthermore, for decreasing time steps $\Delta t$, the width of the wall region also decreases and thus its contribution to the global error will decrease. Combining these results gives that the global discretization error will be $O(\Delta t)$. This will be the case for any function $f(X)$, and therefore the algorithm is $O(\Delta t)$ accurate in the weak sense.

In the remainder of this paper we will focus on the case of simple diffusion, without deterministic forces. Inclusion of a deterministic term, without correction for wall effects, will give an $O(\Delta t)$ global error and, therefore, does neither influence the order of the method nor the essentials of the approach. In the discussion section we will return to this point. For simple diffusion Eq. (1) can thus be simplified to

$$
d X=\sqrt{2 D} d W .
$$

We will further assume here that the wall is located at $x$ $=0$ and that, at the start of the time step under consideration, the particle is located at $x_{0}$, which we take to be on the positive $x$ axis.

In order to find the proper discretization, we first solve the Fokker-Planck equation, equivalent to Eq. (9) near the wall, including the chosen boundary conditions. This gives the spatial distribution function of the displacement of the particle in one time step. Then we determine the zeroth, first, and second moments of that spatial distribution function. Using these moments, one can write the $O(\Delta t)$ discretization for the corresponding stochastic differential equation, using the results of Eqs. (4) and (6).

The well-known solution for simple diffusion in free space, starting at $x_{0}$, over a time interval $\Delta t$, is given by a Gaussian that broadens over time,

$$
p_{\text {free }}\left(\Delta t, x_{0}, x\right)=\frac{1}{2 \sqrt{\pi D \Delta t}} \exp \left[-\frac{\left(x-x_{0}\right)^{2}}{4 D \Delta t}\right]
$$

This solution can be easily adapted to find the solutions for a diffusing particle near a wall, for both wall types, absorbing and reflecting.

To model the influence of the wall on the stochastic motion we propose a survival probability per time step (of course, only relevant for the absorbing wall, otherwise this probability equals 1 )

$$
f_{0}\left(\frac{X}{\sqrt{D \Delta t}}\right)
$$

and, if the particle is not annihilated, a stochastic move of the form

$$
\Delta X=f_{1}\left(\frac{X}{\sqrt{D \Delta t}}\right) \sqrt{D \Delta t}+f_{2}\left(\frac{X}{\sqrt{D \Delta t}}\right) \sqrt{D} \Delta U .
$$

The functions $f_{0}, f_{1}$, and $f_{2}$ can be easily related to the zeroth, first, and second moments of the displacement of a particle in one time step,

$$
\begin{gathered}
f_{0}\left(\frac{x_{0}}{\sqrt{D \Delta t}}\right)=\left\langle x^{0}\right\rangle, \\
f_{1}\left(\frac{x_{0}}{\sqrt{D \Delta t}}\right)=\frac{1}{\sqrt{D \Delta t}}\left[\frac{\langle x\rangle}{\left\langle x^{0}\right\rangle}-x_{0}\right], \\
f_{2}\left(\frac{x_{0}}{\sqrt{D \Delta t}}\right)=\frac{1}{\sqrt{D \Delta t}} \sqrt{\frac{\left\langle x^{2}\right\rangle}{\left\langle x^{0}\right\rangle}-\left[\frac{\langle x\rangle}{\left\langle x^{0}\right\rangle}\right]^{2}} .
\end{gathered}
$$


Here $\left\langle x^{n}\right\rangle$ denote the moments of the appropriate probability density $p\left(\Delta t, x_{0}, x\right)$ for a particle that starts at position $x_{0}$, and is located at a position $x$ a time $\Delta t$ later. If there exists a probability for the particle to be absorbed, the zeroth moment, i.e., the survival probability, $\left\langle x^{0}\right\rangle$, will be smaller than one. To compute the mean displacement and the deviation of the displacement, for particles that survive the time step one needs to use the normalized probability distribution $p\left(\Delta t, x_{0}, x\right) /\left\langle x^{0}\right\rangle$. This explains the $\left\langle x^{0}\right\rangle$ in the denominators of Eq. (13). The expression $f_{1}$ denotes the mean displacement, normalized in a suitable way with $\sqrt{D \Delta t}$, and $f_{2}$ is the normalized square root of the variance of the possible displacements.

The functions $f_{0}, f_{1}$, and $f_{2}$ can be determined using known analytical expressions, but other means of determining the correct (approximate) values for the moments of the displacements are just as good. This can be a numerical evaluation of integrals, but also a Brownian dynamics simulation focused on the region near the wall. In many cases the results of such approaches can be expressed in terms of interpolation functions, which can then be used for a large scale simulation. Moreover, the method is not restricted to pure diffusion as treated here, and different boundary conditions and effects such as curvature can be incorporated. In the following we apply this recipe to the cases of the absorbing and reflecting flat wall. In a forthcoming paper we treat the spherical boundary.

\section{A. The absorbing wall}

For the absorbing wall the analytical solution for the probability density for the final position $x$, reached after a time step $\Delta t$ having started from $x_{0}$, can be written as the superposition,

$$
p_{\text {absorb }}\left(\Delta t, x_{0}, x\right)=p_{\text {free }}\left(\Delta t, x_{0}, x\right)-p_{\text {free }}\left(\Delta t, x_{0},-x\right) .
$$

This solution obeys the correct diffusion equation, the initial condition, and the boundary condition $p_{\text {absorb }}\left(\Delta t, x_{0}, 0\right)=0$. The zeroth, first, and second moments of this distribution are

$$
\begin{gathered}
\left\langle x^{0}\right\rangle=\operatorname{erf}\left(\frac{x_{0}}{\sqrt{4 D \Delta t}}\right), \\
\left\langle x^{1}\right\rangle=x_{0}, \\
\left\langle x^{2}\right\rangle=\left(x_{0}^{2}+2 D \Delta t\right) \operatorname{erf}\left(\frac{x_{0}}{\sqrt{4 D \Delta t}}\right) \\
+\sqrt{\frac{4 D \Delta t}{\pi}} x_{0} \exp \left(-\frac{x_{0}^{2}}{4 D \Delta t}\right) .
\end{gathered}
$$

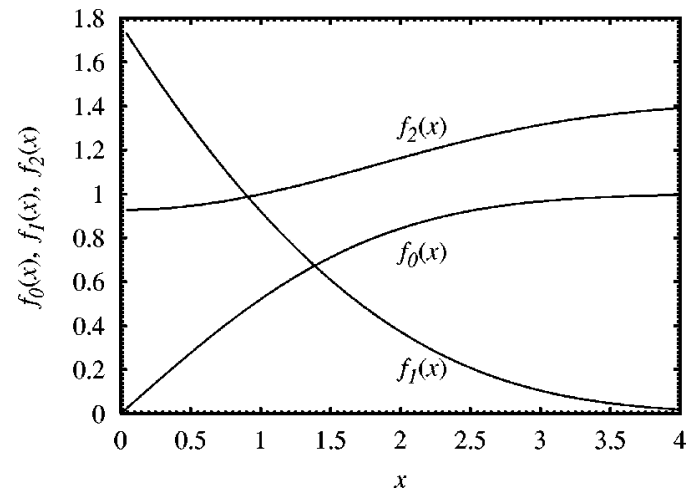

FIG. 1. The survival probability $f_{0}$, the dimensionless mean displacement $f_{1}$, and the dimensionless root-mean-square displacement $f_{2}$ versus the dimensionless initial distance from the wall for the absorbing boundary condition.

Using these moments, the explicit expressions for $f_{0}, f_{1}$, and $f_{2}$ for the case of an absorbing wall are found to be

$$
\begin{gathered}
f_{0}(x)=\operatorname{erf}(x / 2), \\
f_{1}(x)=x \frac{1-\operatorname{erf}(x / 2)}{\operatorname{erf}(x / 2)}, \\
f_{2}(x)=\sqrt{2+x \frac{2}{\sqrt{\pi}} \frac{\exp \left(-x^{2} / 4\right)}{\operatorname{erf}(x / 2)}+x^{2} \frac{\operatorname{erf}^{2}(x / 2)-1}{\operatorname{erf}^{2}(x / 2)} .}
\end{gathered}
$$

The functions $f_{0}, f_{1}$, and $f_{2}$ are plotted in Fig. 1. Equation (12) shows [combined with Eq. (16)] that the influence of the wall becomes important for distances of a few $\sqrt{D \Delta t}$ units from the wall. The wall interaction gives rise to a deterministic contribution of order $\sqrt{D \Delta t}$ and also the stochastic term is affected.

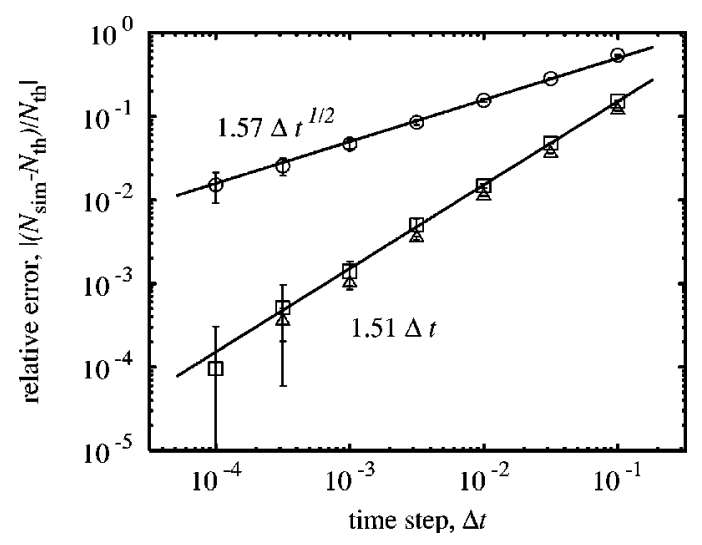

FIG. 2. The relative error in the stationary value of the number of particles in a one-dimensional box with two absorbing walls and a continuous source in the middle. The circles denote a naive implementation; the squares denote our implementation, both using uniformly distributed random processes. The results indicated by the triangles are those generated by directly sampling from a distribution consisting of two equivalent (delta) peaks, having the correct first and second moments. Clearly, our method improves the order of the discretization error from $O(\sqrt{\Delta t})$ to $O(\Delta t)$. 
The problem we use to test our proposed method is that of a one-dimensional box with two absorbing walls. The particles in the box diffuse freely. In the center of the box a continuous source of particles is placed. In Fig. 2 we have plotted the relative error in the mean number of particles present in the box for the stationary case. This system was simulated both by using a naive implementation of the boundary condition and by means of our method. In the naive approach the final position of a particle after a time step is monitored. If this position is at the forbidden side of the wall, the particle is considered to be absorbed during that time step.

In our approach, at the beginning of each time step one determines which particles are absorbed by comparing a random number between 0 and 1 with the survival probability. If the particle survives this operation, a displacement that obeys Eq. (12) is made. This can be done in different ways. One is to use uniformly distributed numbers for $\Delta U$, and directly applying Eq. (12). Another way is by sampling the displacements from a distribution consisting of two possible positions, that are sampled with equal probability, and which gives the correct first and second moments. From Fig. 2 it is clear that our method is superior. The discretization error of the naive implementation is $O(\sqrt{\Delta t})$, while for the improved method we obtained an $O(\Delta t)$ error.

\section{B. The reflecting wall}

The case of a diffusing particle in the neighborhood of a reflecting wall is more simple, but also more subtle in some aspects, than the absorbing case. There is no absorption, so the zeroth moment is equal to 1 and does not change. The analytical solution for the probability density for the final position $x$, reached after a time step $\Delta t$ having started from position $x_{0}$, is given by

$$
p_{\text {reflect }}\left(\Delta t, x_{0}, x\right)=p_{\text {free }}\left(\Delta t, x_{0}, x\right)+p_{\text {free }}\left(\Delta t, x_{0},-x\right),
$$

where $p_{\text {free }}$ is the probability distribution for a free particle, given by Eq. (10). As in the case of the absorbing wall this is a superposition of solutions of the diffusion equation for the correct initial condition. It remains normalized and obeys the boundary condition that the probability flux is zero at the wall, i.e., $\partial p / \partial x=0$ for $x=0$. Using the first and second moments of this distribution one can compute $f_{1}$ and $f_{2}$, similar to the case of the absorbing wall,

$$
\begin{gathered}
f_{1}(x)=\frac{2}{\sqrt{\pi}} \exp \left(-x^{2} / 4\right)-x(1-\operatorname{erf}(x / 2)), \\
f_{2}(x)=\sqrt{2+x^{2}-\left[x \operatorname{erf}(x / 2)+\frac{2}{\sqrt{\pi}} \exp \left(-x^{2} / 4\right)\right]^{2}} .
\end{gathered}
$$

The results are plotted in Fig. 3.

These wall functions can be used to generate stochastic steps using Eq. (12). There is, however, a subtlety that has to be taken into account. When sampling the possible displace-

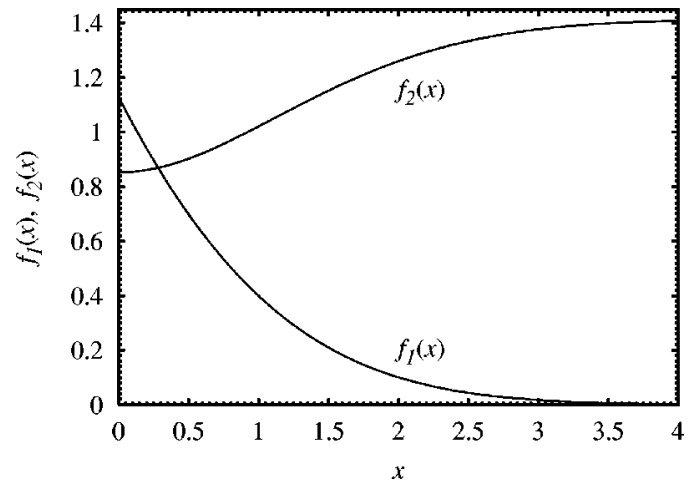

FIG. 3. The dimensionless mean displacement $f_{1}$ and the rootmean-square displacement $f_{2}$ versus the dimensionless initial distance from the wall for the reflecting boundary condition. As no particles disappear, $f_{0}$ is equal to 1 everywhere and therefore not plotted.

ments using the analytic solution, given by Eq. (17), one will never cross the wall. This is not necessarily true for an approximation that only samples the lowest-order moments correctly. When, in this case, one ends up at the forbidden side of the wall, it is not clear how to proceed. One cannot just simply perform a reflection since a reflection also influences the moments of a step. It is therefore best to use a distribution that does not cross the wall. It is important to note that for the reflecting boundary condition the twopeaked distribution obeys this restriction, but the uniform distribution does not. For the uniform distribution the restriction that the wall is not crossed can be expressed by the

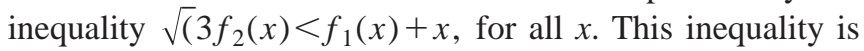

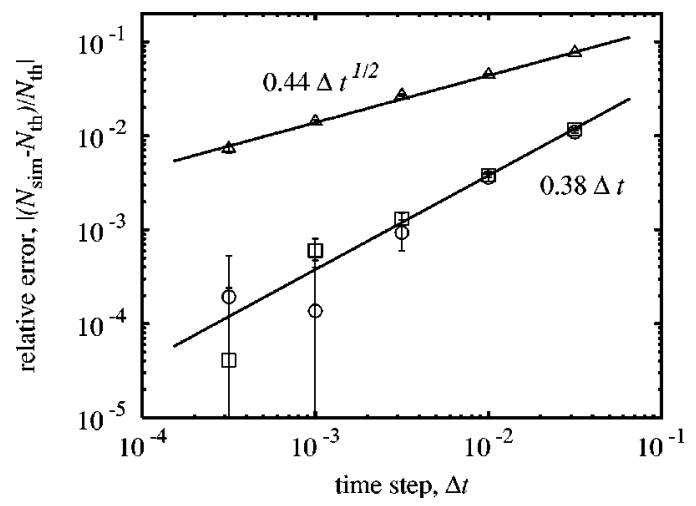

FIG. 4. The relative error in the stationary value of the number of particles in a one-dimensional box with one reflecting wall, and one absorbing wall and a continuous source in the middle. The squares denote the error when using our method for both the reflecting wall and the absorbing wall. At every time step displacements are sampled from a distribution consisting of two equivalent (delta) peaks, their combination having the correct first and second moments. The resulting discretization error is $O(\Delta t)$. Both the circles and the triangles indicate simulations using a naive approach for the reflecting wall. The circles indicate the results for the case where particles are reflected after crossing the reflecting wall (i.e., $X$ $:=-X$ ) for a wall located at $x=0$. The triangles indicate the results when the particle is repositioned on the wall after crossing it (i.e., $X:=0)$. 
obeyed for the wall functions of the absorbing wall, but not for the reflecting wall. So uniformly distributed stochastic displacements cannot be used in this case.

For generating the result in Fig. 4 we therefore used the distribution of two possible positions (sampled with equal probability), having the correct first and second moments, as explained before. The setup of the numerical experiments consists again of two walls with a continuous source of particles in the middle, but now one wall is reflecting and the other one absorbing. The absorbing wall is treated in the same manner as before. Figure 4 shows that the discretization error is $O(\Delta t)$.

In Fig. 4 we also plotted the error in a simulation using the naive approach of simple reflection when the wall is crossed (no wall functions are used). One might be surprised that this simulation also has an $O(\Delta t)$ error and not an $O(\sqrt{\Delta t})$ error. This demonstrates that the demand to sample correct first and second moments is stronger than strictly necessary. (It is, however, a sufficient demand.)

This does not mean that in the case of reflecting boundaries any naive approach will give an $O(\Delta t)$ error. As demonstrated in Fig. 4 the naive approach of repositioning a particle on the wall after it has ended up on the "wrong" side of the wall after a time step, gives rise to an $O(\sqrt{\Delta t})$ error. Of course, for $\Delta t$ approaching zero, the behavior of both cases converges, but the convergence behavior is different. From a physical point of view there is not much reason to prefer one naive approach above the other. In Brownian motion inertia plays no role and, therefore, there is no difference between elastic collisions with the wall and nonelastic collisions. The reason that naive reflection gives more accurate results than reposition the particle at the wall position upon a wall crossing, is that the first procedure preserves the symmetry that is also found in the analytic solution, which is indeed constructed by simple reflection of the solution without wall interaction.

For problems without such a symmetry any naive approach gives large, e.g., $O(\sqrt{\Delta t})$ errors. In that case the safest procedure is therefore to use distributions with correct moments. This always gives rapid convergence. In fact, for systems like many-particle systems that interact in a hard way, our approach might still be preferred, even if the naive approach works. This is because using the naive approach one has to check for violations of boundary conditions. In our approach one only has to detect whether particles are sufficiently close to each other to interact, and then use the appropriate wall functions. This check is much easier to perform.

\section{DISCUSSION AND CONCLUSIONS}

We are aware of two other methods to avoid the large $O(\sqrt{\Delta t})$ discretization error. Both methods have been proposed by Lamm and Schulten [1]. The first method only works for absorbing boundary conditions. Öttinger [2,3] uses a similar method in a problem that is connected to reptation theory. This approach was recently used by Barenbrug et al. [4] to efficiently simulate the diffusion limited chemical reaction between spherical particles. We will now describe it briefly and make a comparison with the method we have introduced here.

The approach is aimed at sampling $p_{\text {absorb }}\left(\Delta t, x_{0}, x\right)$ correctly. The approach consists of two steps. Starting from a position $x_{0}$, a step is made with known statistics $p\left(\Delta t, x_{0}, x\right)$. After this move $p_{\text {absorb }}\left(\Delta t, x_{0}, x\right) / p\left(\Delta t, x_{0}, x\right)$ is taken to be the survival probability. An uniformly distributed random number between 0 and 1 is drawn, if it is larger than $p_{\text {absorb }} / p$ the particle is assumed to be absorbed during this time step. In this way $p_{\text {absorb }}$ is sampled correctly.

The method only works if $p_{\text {absorb }} / p$ is less than 1 everywhere (otherwise particles have to be created instead of annihilated). Therefore the approach can only be used effectively for the absorbing-boundary case. For the absorbing boundary this condition is guaranteed to be fulfilled if one uses the probability density for the displacement in free space for $p$. For pure diffusion this means the use of a Gaussian distribution of displacements. When one uses a uniform distribution, the tail of the correct distribution cannot be reconstructed and the scheme will degenerate into an $O(\Delta t)$ accurate scheme.

In the case of more complex geometries the method needs the determination of $p_{\text {absorb }}\left(\Delta t, \boldsymbol{x}_{0}, \boldsymbol{x}\right)$, assuming that $p\left(\Delta t, x_{0}, \boldsymbol{x}\right)$ is known and simple. For example, in the relatively simple case of the interacting spheres this is a function of two relative distances (starting and final) and the angle between them. This is too complicated to put into an interpolation function. In Ref. [4] we were therefore forced to compute the probability density at every time step, for all particle pairs in each other's vicinity, by evaluating a series expression for $p_{a b s o r b}\left(\Delta t, \boldsymbol{x}_{0}, \boldsymbol{x}\right)$, for all specific sets of $\boldsymbol{x}_{0}$ and $\boldsymbol{x}$.

The second method Lamm and Schulten [1] introduced consists, again, of directly sampling the probability density near a boundary $p_{\text {bound }}\left(\Delta t, x_{0}, x\right)$. Similarly to the first approach, much more information is needed than for the approach we introduced in this paper. They consider flat walls only, with several boundary conditions and several complications such as drift factors and nonconstant diffusivity. Their treatment depends heavily on the fact that an analytic expression of $p_{\text {bound }}\left(\Delta t, x_{0}, x\right)$ (or a good analytical approximation) is available. This expression has to be integrated and inverted to be used to sample the statistics correctly. These operations are computationally very expensive if there is no (simple) analytic expression available. This is different from the pure absorbing case where in principle $p_{\text {bound }}\left(\Delta t, x_{0}, x\right)$ can be computed on the spot, such as done in Ref. [4]. For the flat wall the inversion involves the inverse of the complementary error function. An approximation has to be used for this.

The obvious benefit of the Lamm and Schulten approaches is that, for flat walls, they are (almost) exact. This benefit quickly disappears when the situation becomes more complex. For example, Northrup et al. [5] (see also Ref. [6]) consider the interaction of two spherical particles. Since they used analytical expressions for the absorbing flat wall, curvature effects gave an extra order $\Delta t$ error [not $O(\sqrt{\Delta t})$, which forced them to decrease the time step in the vicinity of the sphere. This can be avoided by using a series expression 
for the solution of the diffusion equation around an absorbing sphere [4], but obviously this is a computationally more expensive method.

In our approach we would only need the zeroth, first, and second moments of $p_{\text {absorb }}\left(\Delta t, \boldsymbol{x}_{0}, \boldsymbol{x}\right)$ for every $\boldsymbol{x}_{0}$. These moments are a function of the relative distance of the spheres only and can be easily stored in an interpolation table. Even if (semi) analytic expressions are not available one could perform simulations with small $\Delta t$ of the wall region only to tabulate the moments of the distributions. These tabulated moments could be used (in the form of wall functions $f_{0}, f_{1}$, and $f_{2}$ ) in a more complex simulation using (much) larger time steps. This illustrates the fact that the method is expected to be much more flexible, and applicable to more diverse problems, than all earlier proposed fixes for errors due to wall interaction.

In this paper we have only discussed simple diffusive motion and not the deterministic contribution. The deterministic part $A \Delta t$ can be simply added to the displacement. This approximate treatment will give an order $\Delta t$ discretization error. Because this error only occurs near the wall, it will contribute only $O(\Delta t)$ to the global error, and does not change the overall order of the method. There may, however, be reasons to include a correction term. A reason could be that $A$ is so large that $A \Delta t$ is of similar magnitude as $\sqrt{D} \Delta U$. Then the inaccuracy in the discretization of this term would dominate the error due to wall interaction. Another reason could be that the error is systematic, thus accumulates and becomes significant. This might, for instance, be the case for reactive particles with attractive interactions. Within the proposed method it should be feasible to construct a correction term (particularly because it is expected to be linear in $A$ ). The same arguments apply for the $O(\Delta t)$ errors due to curvature effects.

To conclude, we summarize our main findings. The simple basic idea we use is that to accurately perform a stochastic simulation, the moments of the probability distribution of the displacement of the particle have to be sampled to a specified order of accuracy. To obtain an $O(\Delta t)$ accurate discretization it is sufficient if the zeroth, first, and second moments of the displacement are sampled correctly. The proposed way to account for wall interaction reduces the discretization error from $O(\sqrt{\Delta t})$ to $O(\Delta t)$, if our method is compared to naive treatments of this interaction. The method is not only able to treat absorbing boundary conditions, but also reflecting ones. Further we expect our method to also be applicable to a whole range of "intermediate" boundary conditions, including sticky walls [7]. For systems with more degrees of freedom our method is expected to be much more efficient than all presently existing strategies.

\section{ACKNOWLEDGMENT}

The research of Dr. Peters has been supported by the Royal Netherlands Academy of Arts and Sciences.
[1] G. Lamm and K. Schulten, J. Chem. Phys. 78, 2713 (1983).

[2] H. Öttinger, J. Chem. Phys. 91, 6455 (1989).

[3] H. Öttinger, Stochastic Processes in Polymeric Fluids (Springer-Verlag, Berlin, 1996).

[4] T. Barenbrug, E. Peters, and J. Schieber, J. Chem. Phys. (to be published).
[5] S. Northrup, M. Curvin, S. Allison, and J. McCammon, J. Chem. Phys. 84, 2196 (1986).

[6] S. Allison, J. McCammon, and J. Sines, J. Phys. Chem. 94, 7133 (1990).

[7] E. Peters and T. Barenbrug, Phys. Rev. E 66, 056702 (2002). 